\title{
Urgent Urologic Surgery in Patients with Acute Coronary Syndrome
}

\author{
Tariq F. Al-Shaiji*, Jaffar M. Hussain, Majd Al-Kabbani, Mostafa A. Faty, Ahmed R. El-Nahas, \\ Abdullatif Al-Terki
}

Urology Unit, Department of Surgery, Al-Amiri Hospital, Kuwait City, Kuwait

Email: *tshaiji@gmail.com

How to cite this paper: Al-Shaiji, T.F., Hussain, J.M., Al-Kabbani, M., Faty, M.A., El-Nahas, A.R. and Al-Terki, A. (2022) Urgent Urologic Surgery in Patients with Acute Coronary Syndrome. Journal of Biosciences and Medicines, 10, 29-32. https://doi.org/10.4236/jbm.2022.101004

Received: December 6, 2021

Accepted: January 8, 2022

Published: January 11, 2022

Copyright $\odot 2022$ by author(s) and Scientific Research Publishing Inc. This work is licensed under the Creative Commons Attribution International License (CC BY 4.0).

http://creativecommons.org/licenses/by/4.0/

(c) (i) Open Access

\begin{abstract}
Urgent urologic interventions create a challenging scenario when they occur in patients suffering from concurrent acute coronary syndrome. Herewith we report two patients with this scenario. Case 1, a male patient was admitted with non-ST elevation myocardial infarction in which dual antiplatelet therapy was initiated. He developed symptomatic right obstructive uropathy. Case 2, a male patient developed non-ST elevation myocardial infarction and was commenced on dual antiplatelet therapy. Subsequently, he developed gross hematuria unresponsive to conservative measures. Urgent urological intervention was carried out while on aspirin in both cases with uneventful recovery. Upon coronary angiography, both cases were found to have multi-vessel disease requiring coronary artery by-pass graft later. Due to their concurrent urologic problems the patients described were not optimized fully to undergo coronary angiography. In close collaboration with cardiology, patients with acute coronary syndrome can be carefully selected to undergo urgent urologic interventions prior to coronary angiography/primary coronary intervention.
\end{abstract}

\section{Keywords}

Acute Coronary Syndrome, Urgent Urologic Conditions, Urgent Urologic Intervention

\section{Introduction}

Urologic conditions such as obstructive uropathy or hematuria, that require urgent surgical management create a particularly challenging scenario when they occur in patients that suffer from concurrent acute coronary syndrome (ACS). The particularly challenging subsets of patients are ones in whom coronary an- 
giography (CAG) with primary coronary intervention (PCI) can be deferred but also in whom surgery is considered high risk. A balance must be struck between managing their urologic condition and between the urgent need for CAG/PCI where the latter may preclude the former. The literature has described few cases with successful outcome; however it remains a grey area with logical concerns for both urologist and cardiologist. We present two cases that were successfully managed with urgent urologic surgery and then subsequent management of their cardiac condition. The patients described in the manuscript had given their consent for the case reports to be published.

\section{Cases Presentation}

Case 1: A 50-year-old gentleman was admitted with non-ST elevation myocardial infarction (NSTEMI). Dual antiplatelet therapy (DAPT) as well as medical therapy was initiated. He was also incidentally found to have mildly elevated serum creatinine compared to baseline which was within normal limits and upon evaluation, was found to have multiple obstructing right distal ureteric calculi with the largest measuring 2 centimeters along with right sided hydronephrosis on plain computed tomography of the abdomen and pelvis causing obstructive uropathy. Ureterorenoscopy (URS) with aspirin only was performed and complete stone fragmentation along with ureteral stent placement was undertaken. $\mathrm{He}$ had an uneventful post-operative course and subsequently underwent CAG that revealed multi-vessel disease requiring coronary artery by-pass graft (CABG). His post-operative course from cardiac point of view was unremarkable as well and had the ureteric stent removed after 4 weeks under local anesthesia.

Case 2: An 85-year-old gentleman with multiple medical comorbidities who was admitted with community acquired pneumonia and acute kidney injury. A urethral catheter was inserted to monitor urinary input and output. During his inpatient stay he was diagnosed with NSTEMI and was commenced on DAPT. Following commencement of DAPT, he subsequently developed hematuria unresponsive to conservative measures that required multiple blood transfusions. Flexible cystoscopy revealed a bleeding, enlarged prostate. He underwent transurethral resection of the prostate (TURP) under aspirin. He was kept on bladder irrigation for 2 days when his urine was finally clear and had successful trail of voiding and uneventful recovery. This was soon followed by CAG that revealed multi-vessel disease requiring CABG eventually.

\section{Discussion}

Due to their concurrent urologic problems the patients described were not optimized fully to undergo CAG. So, the challenge of deciding on whether to pursue treatment of their urologic condition or cardiac condition first, presented itself. In both cases, the extent of the lesions was not known prior to surgery, and it was unknown whether they would require PCI with coronary artery stent placement or CABG. The 30-day mortality post CAG with primary coronary in- 
tervention (stenting or otherwise) is high and approaches 30\% [1]. This creates an opportunity to perform urgent surgery with less risk as compared to in the post PCI setting. Furthermore, placement of a coronary stent requires that patients adhere to a strict DAPT regimen to reduce the risk of stent thrombosis and death [2]. Patients taking DAPT are at a heightened risk for severe bleeding post-operatively particularly in procedures such as TURP where one antiplatelet agent is typically stopped prior to the procedure [3] [4]. Despite blood transfusion with an intent to alleviate this hypoxia, blood transfusions with packed red blood cells are paradoxically associated with increased risk of acute myocardial infarction and death [5]. Hemoglobin level thresholds for transfusion vary between 8 to $10 \mathrm{mg} / \mathrm{dL}$ below which transfusion may be considered [5]. Therefore, profuse bleeding results in tissue hypoxia and myocardial ischemia thus necessitating operative management when conservative measures fail. The two patients described were carefully assessed by a cardiologist and deemed amenable to undergo surgery under general anesthesia with significant risk, however, the proposed benefits of undergoing surgery first, as described above, outweighed the benefits of undergoing surgery after the CAG.

\section{Conclusion}

In close collaboration with a cardiologist, patients with ACS can be carefully selected to undergo urgent urologic interventions prior to CAG/PCI and avoid the heightened risk of peri-operative morbidity and mortality that occurs following CAG/PCI.

\section{Ethics Statement}

Consent was obtained from the patients involved is this short case series.

\section{Conflicts of Interest}

The authors declare no conflicts of interest regarding the publication of this paper.

\section{References}

[1] Savonitto, S., Caracciolo, M., Cattaneo, M. and de Servi, S. (2011) Management of Patients with Recently Implanted Coronary Stents on Dual Antiplatelet Therapy Who Need to Undergo Major Surgery. Journal of Thrombosis and Haemostasis, 9, 2133-2142. https://doi.org/10.1111/j.1538-7836.2011.04456.x

[2] Eberli, D., Chassot, P.G., Sulser, T., Samama, C.M., Mantz, J., Delabays, A. and Spahn, D.R. (2010) Urological Surgery and Antiplatelet Drugs after Cardiac and Cerebrovascular Accidents. Journal of Urology, 183, 2128-2136. https://doi.org/10.1016/j.juro.2010.02.2391

[3] Culkin, D.J., Exaire, E.J., Green, D., Soloway, M.S., Gross, A.J., Desai, M.R., White, J.R. and Lightner, D.J. (2014) Anticoagulation and Antiplatelet Therapy in Urological Practice: ICUD/AUA Review Paper. Journal of Urology, 192, 1026-1034. https://doi.org/10.1016/j.juro.2014.04.103 
[4] Naspro, R., Rossini, R., Musumeci, G., Gadda, F. and Pozzo, L.F. (2013) Antiplatelet Therapy in Patients with Coronary Stent Undergoing Urologic Surgery: Is It Still No Man's Land? European Urology, 64, 101-105.

https://doi.org/10.1016/j.eururo.2013.01.026

[5] Moraes, A.A., Chammas, A.Z., Melo Neto, J., Mendes, L.C., Aguiar, Y.S. and Ramos, R.F. (2012) Bleeding in Non-ST-Segment Elevation Acute Coronary Syndrome. Revista Brasileira de Terapia Intensiva, 24, 284-293.

https://doi.org/10.1590/S0103-507X2012000300013 